\title{
Luminal A and luminal B (HER2 negative) subtypes of breast cancer consist of a mixture of tumors with different genotype
}

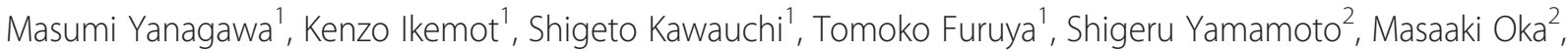 \\ Atunori Oga', Yukiko Nagashima ${ }^{3}$ and Kohsuke Sasaki ${ }^{1 *}$
}

\begin{abstract}
Background: The St Gallen International Expert Consensus 2011 has proposed a new classification system for breast cancer. The purpose of this study was to elucidate the relationship between the breast cancer subtypes determined by the new classification system and genomic characteristics.

Methods: Invasive breast cancers $(n=363)$ were immunohistochemically classified as follows: $111(30.6 \%)$ as luminal A, 95 (26.2\%) as luminal B (HER2 negative), 69 (19.0\%) as luminal B (HER2 positive), 41 (11.3\%) as HER2, and $47(12.9 \%)$ as basal-like subtypes.

Results: The high expression of Ki-67 antigen was detected in 236 tumors; no cases of luminal A subtype showed high expression of the Ki-67 antigen, but more than $85 \%$ of tumors of the other subtypes showed high expression. In addition, DNA ploidy and chromosomal instability (CIN) were assessed using imaging cytometry and FISH, respectively. In this series, 336 (92.6\%) tumors consisted of 129 diploid/CIN- and 207 aneuploid/CIN + tumors. Diploid/CIN- and aneuploid/CIN+ features were detected in $64.9 \%$ and $27.9 \%$ of luminal $\mathrm{A}, 41.1 \%$ and $49.5 \%$ of luminal B (HER2-), $11.6 \%$ and $81.2 \%$ of luminal B (HER2+), $4.9 \%$ and $90.2 \%$ of HER2, and $17.0 \%$ and $76.6 \%$ of basal-like subtypes, respectively. Unlike the luminal B (HER2+), HER2 and basal-like subtypes, the luminal A and luminal B (HER2-) subtypes were heterogeneous in terms of DNA ploidy and CIN.
\end{abstract}

Conclusions: It is reasonable to propose that the luminal A and luminal B (HER2-) subtypes should be further divided into two subgroups, diploid/CIN- and aneuploid/CIN+, based on their underlying genomic status.

\section{Background}

Breast cancer is one of the most common malignant tumors in the world, and a large number of patients die of the disease every year. Morphologically and biologically, breast cancer is a heterogeneous disease family comprising a number of subtypes [1]. Although the conventional histological classification system is indispensable for the accurate histological diagnosis of breast cancer, it does not always provide sufficient information to evaluate the biological characteristics of individual tumors and it is not useful for treatment selection. Indeed, it is well known that tumors with the same histological subtypes can have very different biological trajectories.

\footnotetext{
* Correspondence: kohsuke@yamaguchi-u.ac.jp

'Departments of Pathology, Yamaguchi University Graduate School of Medicine, Ube-shi 755-8505, Japan

Full list of author information is available at the end of the article
}

This situation indicates the need for a more reliable classification system, which guides clinical decision-making such as the determination of an optimal therapeutic strategy for individual cancer patients [2,3]. Determining the status of estrogen and progesterone receptors, HER2 amplification and Ki-67 antigen expression is practical and valuable for estimating the patient prognosis and for determination of the treatment strategy [4]. Recently, the St Gallen International Expert Consensus proposed a new intrinsic biological classification system based on the expression of the estrogen receptor (ER), progesterone receptor (PgR), HER2 and Ki-67 [5]. The classification system categorizes invasive breast carcinomas into the following five distinct molecular subtypes; luminal $\mathrm{A}$, luminal B (HER2-), luminal B (HER2+), HER2, and basallike subtypes, and these subtypes are linked to the therapeutic selection [5]. The classification can be performed

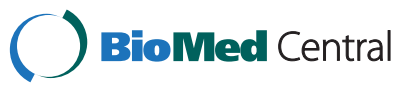


in every pathological laboratory where IHC with a simple panel of markers is possible, and this approach is designated as the IHC-based classification [6,7]. The clinical value of this classification system is still ongoing world wide.

The biological characteristics of a tumor are primarily affected by genomic changes. In this context, the nuclear DNA content has been measured to estimate the biological characteristics of solid tumors including breast cancer, and in general, it is accepted that DNA aneuploid cancers represent a poorer prognosis than diploid tumors [8-13]. However, the new classification system has not been evaluated with regard to the status of DNA ploidy and/or CIN. The relationship between the ICHbased subtypes and the status of DNA ploidy and CIN should be elucidated to better understand the differences in the biological characteristics within and between subtypes as a precondition to achieve personalized treatment for breast cancer, and the ICH-based classification system should be evaluated in terms of the status of DNA ploidy and CIN.

In this study, the DNA ploidy and CIN were compared with the subtypes classified by the IHC-based classification system in 363 consecutive invasive breast cancer patients.

\section{Methods}

\section{Tumor tissue specimens}

This study evaluated 363 primary invasive breast cancers. None of the patients had any family history of hereditary breast cancer. The average age of patients was 56.9 years, ranging from 30 to 87 years old. Patients had received neither chemotherapy nor radiation prior to surgery. The Institutional Review Board for Human Use at Yamaguchi University Graduate School of Medicine approved the study protocol, and informed consent for this study was obtained from all patients. Representative parts of the surgically removed tumor tissues were used for touch-smear preparations before fixation. Tumor tissue specimens were fixed in $10 \%$ formalin overnight and were subjected to histological examinations including the nuclear grade. An immunohistochemical analysis was performed to classify the breast cancers into molecular subtypes (IHC-based subtypes) [5]. The touchsmear preparations were subjected to fluorescence in situ hybridization (FISH) and laser scanning cytometry (LSC) to evaluate the genomic instability status and DNA ploidy, respectively [14].

\section{Immunohistochemistry (IHC)}

The expression status of the ER, PgR, HER2, and Ki-67 antigen was evaluated by an immunohistochemical analysis with antibodies against the ER (1D5, 1:50 dilution, Dako, Denmark), PgR (PR88, no dilution, BioGenex, San
Ramon, CA), and Ki-67 antigen (MIB-1, 1:100 dilution, $\mathrm{DAKO}$ ) in formalin-fixed, paraffin-embedded tissue serial sections as previously described [14]. Prior to immunohistochemical staining, antigen retrieval was performed with microwave heating of tissue sections in a citrate buffer solution at $\mathrm{pH}$ 6.0. A cut-off value of $1 \%$ for both receptors was used to classify the expression of ER and PgR according to criteria proposed by 'The Japanese Society of Breast Cancer' [15] and others [4,16,17]. When immunostaining was observed in more than $1 \%$ of tumor nuclei, the tumor was considered to be positive for the ER or PgR. In brief, breast cancers were classified into five subtypes as follows: luminal A (ER+, PgR+ or PgR-, HER2-, and low Ki-67 index), luminal B (HER2 -) (ER+, PgR+ or PgR-, HER2-, and high Ki-67 index), Luminal B (HER2+) (ER+, PgR + or PgR-, and HER2+), HER2 (ER-, PgR-, and HER2+), and basal-like (ER-, PgR-, and HER2-).

The expression of Ki-67 antigen was scored for the percentage of tumor cell nuclei with positive immunostaining above the background level by observing at least 1000 tumor cell nuclei (Ki-67 index). In this study, the Ki-67 index was scored as high when $14 \%$ or more of the tumor cells were immunostained according to the guidelines of the 'St Gallen International expert Consensus' [5]. All tumors were scored as either high or low according to the Ki-67 index. The immunostained slides were evaluated independently by two of the authors (K. I. and T. F.).

\section{CIN assessed by FISH}

Chromosomal instability (CIN) was examined by FISH using four pericentromeric probes (chromosomes 7, 11, 17, and 18 for D7Z1, D11Z1, D17Z1, and D18Z1, respectively; Abbott Laboratories, Abbott Park, Illinois, IL) on the touch-smear preparations as previously described [18-20], and the presence or absence of CIN was determined according to the degree of variations in the number of FISH spots between nuclei [20-22]. Slides were counterstained with 4'6-diamidino-2-phenylindole (DAPI). CIN was considered to be positive when the fraction of cells with a modal chromosome number was less than $75 \%$ for four chromosomes [18-22]. HER2 amplification was tested on the smear preparations for IHC equivocal cases using a PathVysion HER2 DNA Probe Kit (Abbott Laboratories) according to the manufacturer's instructions as described previously. A tumor was considered to be as positive for HER2 gene amplification when the HER2/CEP 17 ratio was 2.2 or higher.

\section{Determination of DNA ploidy by LSC}

Measurement of the nuclear DNA content by LSC was performed as described previously [20,21]. Briefly, the touch-smear preparations fixed in $70 \%$ ethanol were 
dipped in a propidium iodide solution $(25 \mu \mathrm{g} / \mathrm{ml}$ in PBS) containing 0.1\% RNase (Sigma-Aldrich Co., St Louis, $\mathrm{MO})$. The DNA content was measured by a laser scanning cytometer (LSC 101; Olympus). Usually, more than 5,000 cells were examined in each sample. A DNA histogram was generated, and the DNA ploidy was determined. DNA ploidy was expressed as the DNA index (DI). A case with $1.0 \leq \mathrm{DI}<1.2$ was classified as a diploid and all others were classified as aneuploid tumors.

\section{Statistical analysis}

The differences in the frequency of marker expression frequency between two groups were determined using the Chi-square test. A difference was considered to be significant for P-values $<0.05$.

\section{Results}

\section{IHC-based classification}

Of the 363 breast cancers, 258 (71.1\%) were ER positive and 218 (60.1\%) were PgR positive. In this series, 47 (12.9\%) tumors were triple-negative (ER and PgR negative expression and no HER2 amplification). The expression of the Ki-67 antigen was deemed positive in 237 (65.3\%) tumors (Ki-67+) (Figure 1). HER2 was positive in $110(30.3 \%)$ tumors, which were classified into either the luminal B or HER2 subtype.

\section{IHC-based subtype classification}

According to the recent criteria [5], breast cancers were classified into five IHC- based subtypes as follows: 111 (30.6\%) as the luminal A subtype, 95 (26.2\%) as the luminal B (HER2-) subtype, 69 (19.0\%) as the luminal B (HER2+) subtype, 41 (11.3\%) as the HER2 subtype, and 47 (12.9\%) for as basal-like (Table 1).

\section{IHC-based subtypes and Ki-67 antigen expression}

The high expression of Ki-67 antigen was detected in 237 (65.3\%) tumors: 0 (0\%) of the luminal A tumors, 95 (100\%) of the luminal B (HER2-) tumors, 59 (85.5\%) of the luminal B (HER2+) tumors, 40 (97.6\%) of the HER2 tumors, and 43 (91.5\%) of the basal-like tumors (Table 1$)$. The average Ki-67 index was 5.8\% ( \pm 3.6 standard deviation) for the luminal A subtype, 24.3\% ( \pm 9.8$)$ for the luminal B (HER2-) subtype, 31.0\% $( \pm 15.8)$ for the luminal B (HER2+) subtype, $43.0 \%( \pm 19.6)$ for the HER2 subtype, and $46.4 \%( \pm 23.1)$ for the basal-like subtype. The average Ki-67 index was significantly different between the luminal $\mathrm{A}$ and the other subtypes $\left(\mathrm{P}=7.366 \times 10^{-12}\right.$, $\mathrm{P}=4.662 \times 10^{-12}, \mathrm{P}=4.061 \times 10^{-10}$, and $\mathrm{P}=7.779 \times 10^{-4}$ between the luminal A subtype and luminal B (HER2- \& HER2+), HER2, and basal-like subtypes, respectively) (Figure 2).

\section{DNA ploidy}

The DNA indices (DIs) ranged from 1.0 to 3.34 in this series of breast cancers. According to the DIs, the 363 breast cancers were divided into two groups, 144 diploid $(1.0 \leq \mathrm{DI}<1.2)$ and 219 aneuploid (DI $\geq 1.2)$ tumors.

\section{Chromosomal instability (CIN)}

In this series, 140 (38.6\%) tumors were classified as CIN negative (CIN-), and 223 (61.4\%) were classified as CIN positive $(\mathrm{CIN}+)$ according to the size of the variant fraction in the chromosome copy number. In this series, $92.8 \%$ of the $223 \mathrm{CIN}+$ tumors were aneuploid, and $94.5 \%$ of the 219 aneuploid tumors were CIN+. In contrast, $92.1 \%$ of the $140 \mathrm{CIN}$ - tumors were diploid, and $89.6 \%$ of the 144 diploid tumors were CIN-.

\section{DNA ploidy and CIN}

Diploid/CIN- features were detected in 129 (35.5\%) of breast tumors and aneuploid/CIN- features were detected in 207 (57.0\%) tumors, respectively. In this study, 92.6\% of the breast cancers were divided into two groups, 129 diploid/CIN- and 207 aneuploid/CIN+ tumors.

\section{IHC-based subtypes, DNA ploidy and CIN}

The diploid/CIN- status was detected in 72 (64.9\%) of the 111 luminal A carcinomas, 39 (41.1\%) of the 95 luminal B (HER2-) carcinomas, 8 (11.6\%) of the 69 luminal B (HER2+) carcinomas, 2 (4.9\%) of the 41 HER2 carcinomas, and $8(17.0 \%)$ of the 26 basal-like carcinomas. The frequency of diploid/CIN- tumors was higher in the luminal A carcinomas than in luminal B (HER2-), luminal B (HER2+), HER2, and basal-like carcinomas $\left(\mathrm{P}=6.315 \times 10^{-4}, \mathrm{P}=2.690 \times 10^{-12}, \mathrm{P}=5.133 \times 10^{-11}\right.$, and $\mathrm{P}=$ $3.823 \times 10^{-8}$ between luminal A subtype and luminal $\mathrm{B}$ (HER2-), luminal B (HER2+), HER2, and basal-like subtypes, respectively) (Figure 3 ). In contrast, the aneuploid/CIN+ status was detected in $31(27.9 \%)$ of the 111 luminal A, 47 (49.5\%) of the luminal B (HER2-), 56 (81.2\%) of the 69 luminal B (HER2+), 37 (90.2\%) of 41 HER2, and $36(76.6 \%)$ of the 47 basal-like subtype tumors. The frequency of aneuploid/CIN+ tumors was lower in luminal A subtypes than in luminal B (HER2-), luminal $\mathrm{B}(\mathrm{HER} 2+)$, HER2, and basal-like subtypes $(\mathrm{P}=$ $1.482 \times 10^{-3}, \mathrm{P}=3.962 \times 10^{-12}, \mathrm{P}=6.996 \times 10^{-11}, \mathrm{P}=1.525 \times 10^{-8}$ between luminal A subtype and luminal B (HER2-), HER2 (HER2+), HER2, and basal-like subtypes, respectively) (Figure 3).

In this series, $41.1 \%$ and $49.5 \%$ of luminal B (HER2-) subtype cases showed diploid/CIN- and aneuploid/CIN+ features , respectively. The proportion of diploid/CINand aneuploid/CIN+ in luminal B (HER2-) was similar to that in all tumors, in which the percentage of diploid/CINand aneuploid/CIN+ was $35.5 \%$ and $57.0 \%$, respectively 


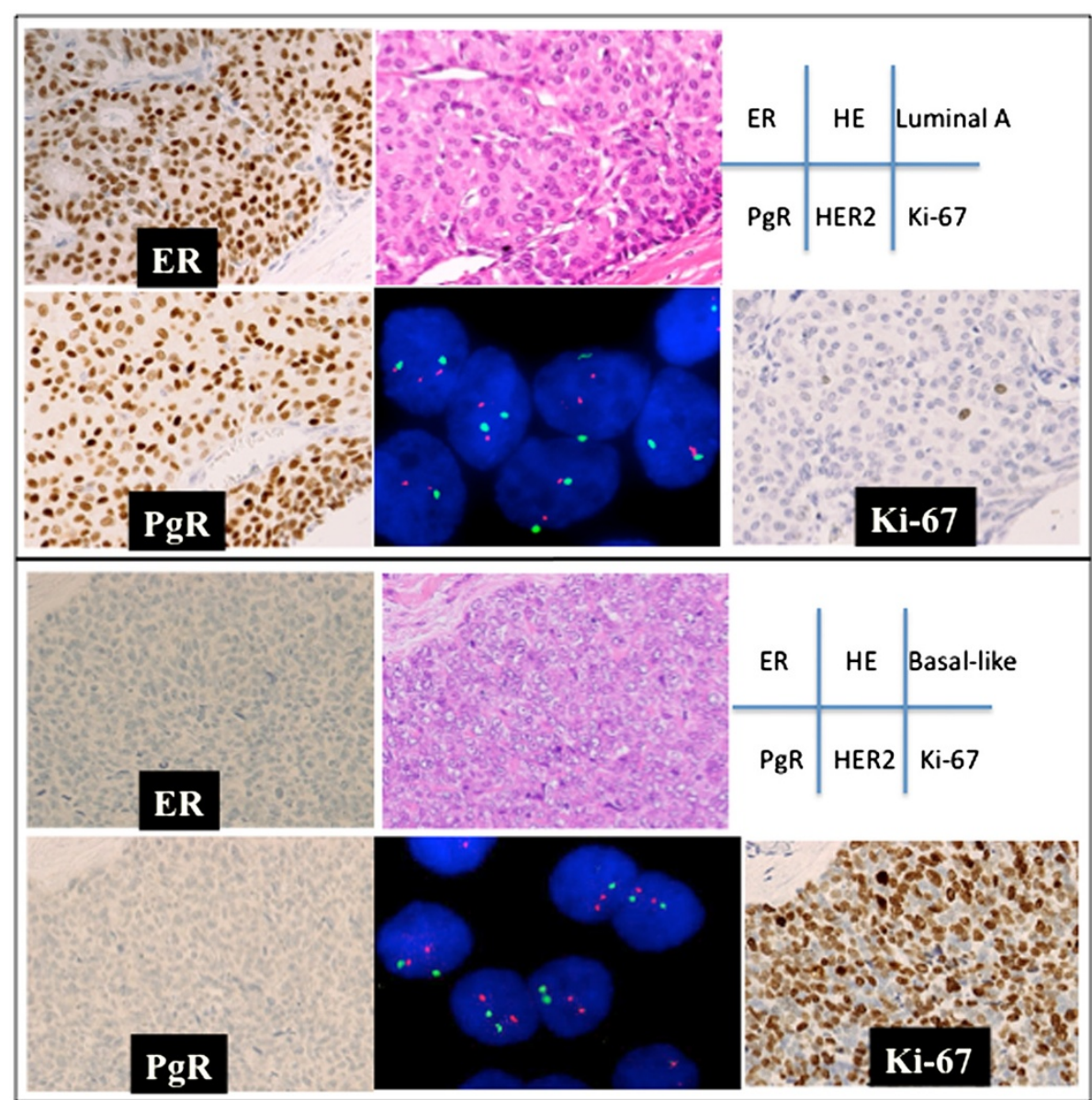

Figure 1 The results of the immunohistochemical analysis evaluating the expression status of the ER, PgR, and Ki-67 antigens, FISH detecting HER2 amplification, and the histological features in conventional tissue sections stained with hematoxylin-eosin. Upper rows: A case of invasive ductal carcinoma classified into the luminal A subtype. The ER was expressed in 100\% of the tumor cells, and the PgR was expressed in 90\% of them. No HER2 amplification was detected (green and red spots indicate chromosome 17 centromeres and HER2). The Ki-67 antigen was expressed in $1 \%$ of the cells in this tumor (Ki-67 index $=1 \%)$. Lower rows: A medullary carcinoma was classified into the basal-like subtype. The ER and PgR are apparently not expressed in any of the tumor cells. No HER2 amplification was detected. The Ki-67 antigen was expressed in $70 \%$ of the tumor cells (Ki-67 index $=70 \%$ ).

(Figure 3). The diploid/CIN+ and aneuploid/CIN- classifications were very rare.

\section{IHC-based subtypes, grade, and DNA ploidy/CIN}

This breast cancer series included 78 grade1, 69 grade 2, and 206 grade 3 tumors. Grade 1 tumors were detected in $47.7 \%$ of the 111 luminal A subtype tumors, $15.8 \%$ of the 95 luminal B (HER2-) subtype tumors, $8.7 \%$ of the 69 luminal B (HER2+) subtype tumors, $0 \%$ of the 41 HER2 subtype tumors, and $8.5 \%$ of the 47 basal-like subtype tumors (Figure 4). Of the grade1 tumors 67.9\% were categorized as being the luminal A subtype. Grade 3 tumors were detected in $18.0 \%$ of luminal A subtype tumors, $58.9 \%$ of luminal B (HER2-), $75.4 \%$ of luminal B (HER2+) subtypes, 92.7\% of HER2 subtypes, and 85.1\% of the basal-like subtype tumors (Figure 4).
Grade 1 tumors were detected in 54 (41.9\%) of the 129 diploid/CIN- cancers and 19 (9.2\%) of the 207 aneuploid/CIN+ cancers. In contrast, grade 3 tumors were detected in $35(27.1 \%)$ of the diploid/CIN- cancers and $161(77.8 \%)$ of the aneuploid/CIN + cancers. Diploid/ CIN- and aneuploid/CIN+ features were found in $69.2 \%$ and $24.4 \%$ of grade 1 tumors, respectively. Diploid/CINand aneuploid/CIN+ features were found in $17.0 \%$ and $78.2 \%$ of grade 3 tumors, respectively (Figure 5 ).

\section{Discussion}

Recently, a molecular classification system was proposed to categorize breast cancers into subtypes associated with the optimal therapeutic modality, and it has become widely used [5]. In this study, the genomic status together with the cell proliferation activity was compared between IHC-based subtypes. 
Table 1 The status of genomic and cell proliferation markers in the ICH-based subtypes

\begin{tabular}{lcccccc}
\hline Markers & LumWalA & Luminal B, HER2- & Luminal B, HER2+ & HER2 & Basal-hke & Total \\
\hline No. of tumors & $111(30.6 \%)$ & $95(26.2 \%)$ & $69(19.0 \%)$ & $41(11.3 \%)$ & $47(12.9 \%)$ & 363 \\
Age (range) & $58.6(34-84)$ & $54.8(27-84)$ & $54.7(30-78)$ & $58,9(38-87)$ & $56.8(31-84)$ & 56.7 \\
Ki-67c14\% & $111(100 \%)$ & $0(0 \%)$ & $10(14.5 \%)$ & $1(24.3 \%)$ & $4(8.5 \%)$ & $126(34.7 \%)$ \\
K6714\% & $0(0 \%)$ & $95(100 \%)$ & $59(85.5 \%)$ & $40(97.8 \%)$ & $43(91.5 \%)$ & $237(65.3 \%)$ \\
DiploEd & $78(70.3 \%)$ & $44(46.3 \%)$ & $11(15.9 \%)$ & $3(7.3 \%)$ & $8(17.0 \%)$ & $140(38.6 \%)$ \\
Aneuploid & $33(29.7 \%)$ & $51(53.7 \%)$ & $58(84.1 \%)$ & $38(92.7 \%)$ & $39(83.0 \%)$ & $223(61.4 \%)$ \\
CIN- & $74(66.7 \%)$ & $43(45.3 \%)$ & $10(14.5 \%)$ & $3(7.3 \%)$ & $10(21.3 \%)$ & $140(38.6 \%)$ \\
CIN. & $37(33.3 \%)$ & $52(54.7 \%)$ & $59(85.5 \%)$ & $38(92.7 \%)$ & $37(787 \%)$ & $223(61.4 \%)$ \\
Diplod!CIN- & $72(64.9)$ & $39(41.1 \%)$ & $8(11.6 \%)$ & $2(4.9 \%)$ & $8(17.0 \%)$ & $129(35.5 \%)$ \\
AneuplodiCIN+ & $31(27.9 \%)$ & $47(49.5 \%)$ & $56(81.2 \%)$ & $372(90.2 \%)$ & $36(76.6 \%)$ & $207(57.0 \%)$ \\
Grade 1 & $53(47.7 \%)$ & $15(15.8 \%)$ & $6(8.7 \%)$ & $2(0 \%)$ & $4(8.5 \%)$ & $78(20.4 \%)$ \\
Grade 2 & $32(28.8 \%)$ & $23(24.2 \%)$ & $10(14.5 \%)$ & $38(4.9 \%)$ & $2(4.3 \%)$ & $69(19.0 \%)$ \\
Grade 3 & $20(18.0 \%)$ & $56(58.9 \%)$ & 1 & 1 & $40(85.1 \%)$ & $206(57.3 \%)$ \\
nd & 6 & 1 & 1 & 1 & 10
\end{tabular}

The numbers in the table indicate the number of tumors fitting each item. The numbers in parenthesis are the percentages of tumors fitting each hem. Ki-67+; tumors with a high Ki-67 index, Ki-67-; tumors with a ow Ki-67 index, CIN-; the absence of chromosomal instability, CIN+; the presence of chromosomal instability, Dip/CIN-IKi-67+; tumors with diploidiCIN -IKi-67- features, Aneup/CIN $\div / \mathrm{Ki}-67 \div$; tumors.

Although the optimal threshold of the Ki-67 index has been a matter of controversy because of interlaboratory variations, the Ki-67 index has been used to divide breast cancers into tumors with low and high proliferation activity $[5,16,23,24]$. The expression level of the Ki-

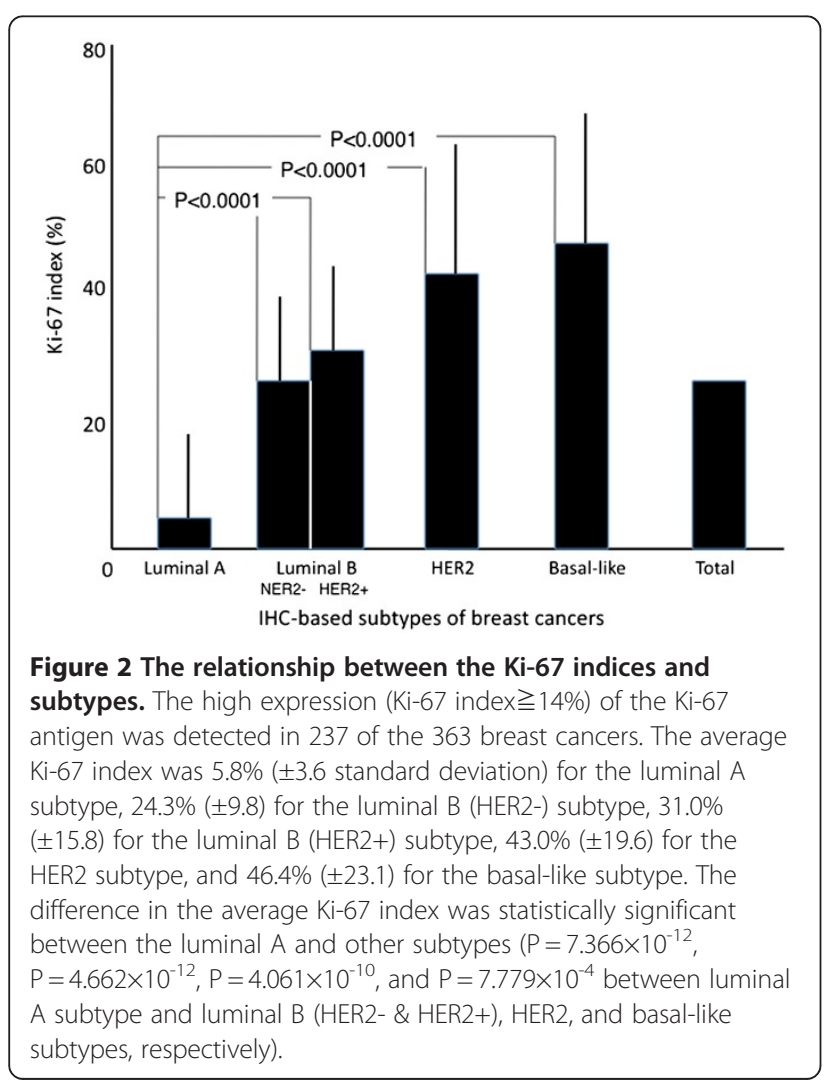

67 antigen was generally connected with the IHC-based subtypes [25]. Not surprisingly, all luminal A subtype tumors showed a low Ki-67 index. In contrast, more than $85 \%$ of luminal B, HER2 and basal-like subtypes had a high Ki-67 index. It has been suggested that the cell proliferation activity is much lower in luminal A tumors than in other subtypes. The cell proliferation activity as well as other cellular characteristics such as the HER2 expression is a useful marker for categorizing breast cancer, and it is primarily affected by the genomic status.

Determination of DNA ploidy in individual tumors is a simple method to examine crude changes in the genome, but it is very informative. Although aneuploidy and CIN phenotype are different by definition [26,27], almost all aneuploid breast cancers in the present study displayed CIN + features and vice versa in agreement with previous reports [20,21,27,28]. Smid and colleagues reported that in particular the basal-like subtype showed the CIN+ feature [28]. The majority (93.4\%) of breast cancers were divided into either diploid/CIN- or aneuploid/CIN+ tumors. The aneuploid/CIN+ status was the major underlying phenotype in the luminal B (HER2+), HER2, and basal-like subtypes. Approximately $80 \%$ of tumors with diploid/CIN- features were of either the luminal A or luminal B (HER2-) subtypes, and a considerable number of aneuploid/CIN+ tumors were also included in these two subtypes. These observations indicate that luminal A and luminal B (HER2-) subtypes can be differentiated from other subtypes based on their underlying genomic changes.

The genomic changes detected by array-based CGH were obviously different between diploid/CIN- and 


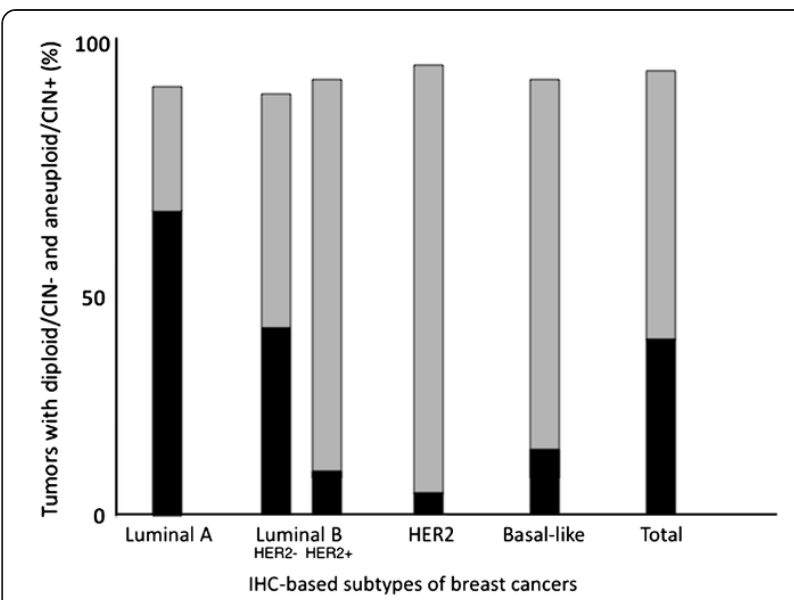

Figure 3 The relationship of subtypes to the DNA ploidy and CIN. The diploid/CIN- status was detected in 72 (64.9\%) of the 111 luminal A carcinomas, 39 (41.1\%) of the 95 luminal B (HER2-) carcinomas, 8 (11.6\%) of the 69 lumminal B (HER2+) carcinomas, 2 (4.9\%) of the 41 HER2 carcinomas, and 8 (17.0\%) of the 26 basal-like carcinomas. In contrast, the aneuploid/CIN + status was detected in 31 (27.9\%) of the 111 luminal A, 47 (49.5\%) of the luminal B (HER2-), $56(81.2 \%)$ of the 69 luminal B (HER2+), 37 (90.2\%) of the 41 HER2, and $36(86.6 \%)$ of the 47 basal-like subtype tumors. The incidence of diploid/CIN- and aneuploid/CIN+ status was different between the luminal A subtype and luminal B (HER2-), luminal B (HER2+), HER2, and basal-like subtypes $(p=0.0006, p=5 E-13, p=5 E 10-12$, and $\mathrm{p}=8 \mathrm{E} 10-9$ ). In addition, the incidence of diploid/CIN- and aneuploid/CIN+ status was statistically different between the luminal B (HER2-) subtype and luminal B (HER2+), HER2, and basal-like subtypes ( $p=0.00002, p=0.000009$, and $p=0.002$ ). Black bar; diploid/CIN- tumors, gray bar; aneuploid/CIN+ tumors, white column; others.

aneuploid/CIN + breast cancers, as previously reported [28]. DNA ploidy and the CIN status are linked to the cell proliferation activity [29-31]. In addition, aneuploid and $\mathrm{CIN}+$ features are associated with a high proliferation activity and poor prognosis when compared to diploid and CIN- tumors [32]. The DNA ploidy and CIN status were different between luminal B (HER2+), HER2, and basal-like subtypes and the remaining two subtypes. As mentioned above, more than $75 \%$ of luminal B (HER2+), HER2 and basal-like subtypes showed aneuploid/CIN + features . In contrast, $41.1 \%$ and $49.5 \%$ of the luminal B (HER2-) subtype showed diploid/CINand aneuploid/ CIN+ features, respectively. The proportion of diploid/CIN- and aneuploid/CIN + tumors in the luminal B (HER2-) subtype was similar to that in all tumors. This similarity proves that the luminal B (HER2-) subtype can be further divided into subgroups based on the underlying genomic status. Thus, both luminal A and luminal B (HER2-) subtypes can be divided into two subgroups, diploid/CIN- and aneuploid/CIN+, based on the genomic characteristics of the tumors. Since aneuploid and/or CIN+ tumors show a poorer prognosis $[8-13,33,34]$, the further classification of luminal $\mathrm{A}$ and

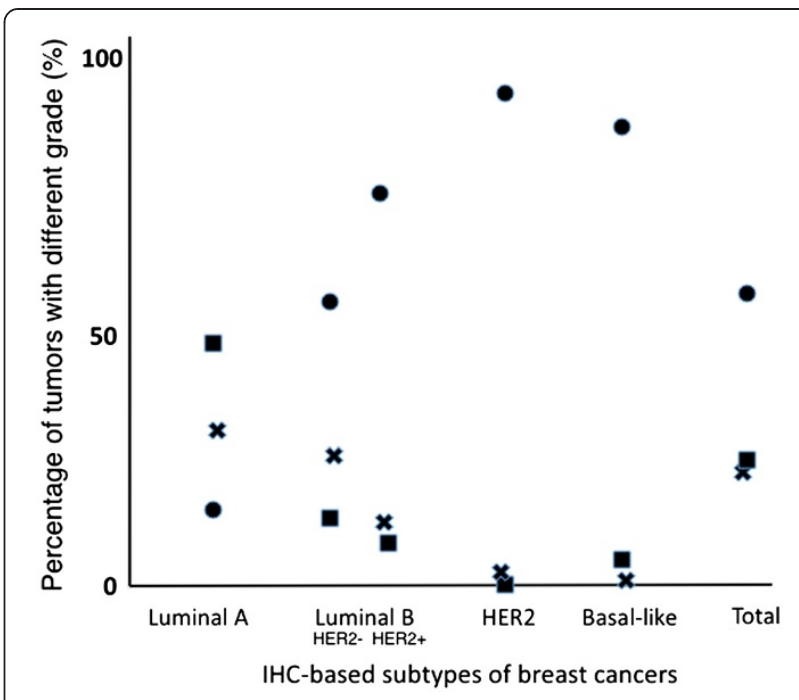

Figure 4 The relationship between the nuclear grade and subtypes. Grade 1 tumors were detected in $47.7 \%$ of the 111 luminal A subtype tumors, $15.8 \%$ of the 95 luminal B (HER2-) subtypes, $8.7 \%$ of the 69 luminal B (HER2+) subtypes, $0 \%$ of the 41 HER2 subtypes, and $8.5 \%$ of the 47 basal-like subtype tumors. Of the grade1 tumors $67.9 \%$ were categorized as part of the luminal A subtype. Grade 3 tumors were detected in 18.0\% of luminal A tumors, $58.9 \%$ of luminal B (HER2-), 75.4\% of luminal B (HER2+), $92.7 \%$ of HER2, and $85.1 \%$ of basal-like tumors. In the HER2 and basal-like subtypes, grade3 tumors were common but grade 1 tumors are rare. In contrast, the luminal A and luminal B (HER2-) subtypes consisted of heterogeneous populations with different grade. Solid square; grade 1 tumors, cross; grade 2 tumors, and solid circle; grade 3 tumors.

luminal B (HER2-) subtypes may be useful for estimating the prognosis of patients with breast cancer and for clinical decision-making. Further studies are necessary to evaluate the additional classification based on these features. The relationship between CIN and patient prognosis depends on the status of ER expression in breast cancers, paradoxical relationship between CIN and patient prognosis in ER- breast cancers was recently reported [35,36]. Luminal A and luminal B (HER2-) subtypes, which were focused in this study, are ER+ by definition.

The conventional nuclear grading system allows for a rough estimation of the prognosis for breast cancer patients [37]. The nuclear grade of tumor cells can be used as a surrogate marker of the Ki-67 index in the new classification system [5]. In this series, more than $85 \%$ of the HER 2 and basal-like subtype tumors represented features of grade 3 cancer, whereas the luminal A and luminal B (HER2-) subtypes consisted of mixture of tumors with different grades. The nuclear grade was linked with the DNA ploidy and CIN status. It could be said that the luminal A and luminal B (HER2-) subtypes were heterogeneous both genomically as well as phenotypically. Thus, it is therefore reasonable to propose that the luminal A and luminal B (HER2-) subtypes should 


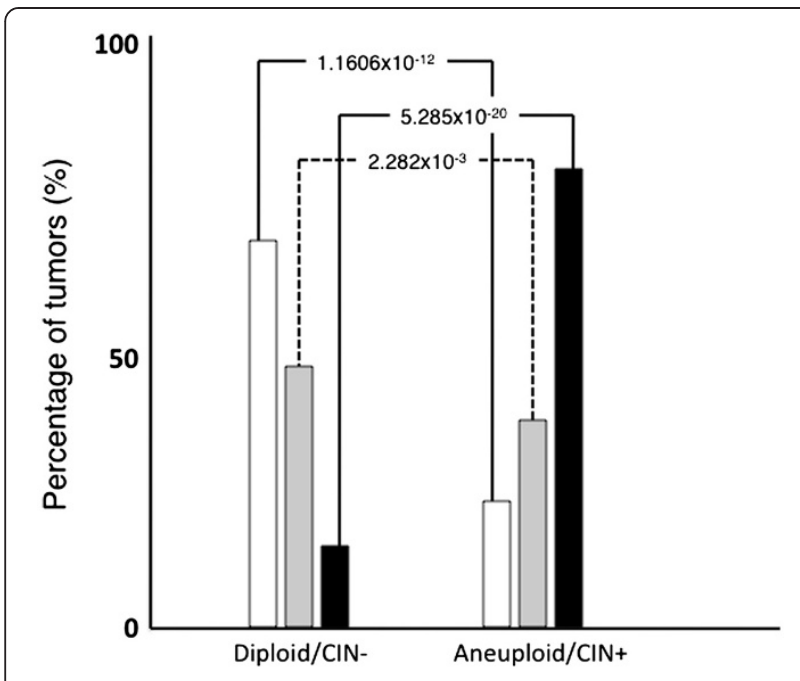

Figure 5 The differences in the nuclear grades between diploid/CIN- and aneuploid/CIN+ breast cancers. Grade 1 tumors were more frequently seen in diploid/CIN- carcinomas than in aneuploid/CIN+ carcinomas $\left(p=1.601 \times 10^{-12}\right)$. In contrast, grade 3 tumors were much more common in aneuploid/CIN+ tumors than in diploid/CIN- tumors $\left(p=5.285 \times 10^{-20}\right)$. The incidence of grade 2 tumors was significantly different between these two types of tumors with different genomic characteristics $\left(P=2.281 \times 10^{-3}\right)$. White column; grade 1 tumors, gray column; grade 2 tumors, and black column; grade 2 tumors.

be further divided into two subgroups, diploid/CIN- and aneuploid/CIN+, based on their underlying genomic status.

\section{Conclusions}

Unlike the luminal B (HER2+), HER2 and basal-like subtypes, the luminal A and luminal B (HER2-) subtypes were heterogeneous in terms of DNA ploidy and CIN. Thus, it is reasonable to propose that the luminal $\mathrm{A}$ and luminal B (HER2-) subtypes should be further divided into two subgroups, diploid/CIN- and aneuploid/CIN+, based on their underlying genomic status.

\section{Abbreviations \\ ER: Estrogen receptor; PgR: Progesterone receptor; CIN: Chromosomal instability.}

\section{Competing interests}

The authors declare no competing interest.

\section{Acknowledgments}

This work was supported in part by The Ministry of Education, Culture of Japan (20659055 and 21659088), The New Energy and Industrial Technology Development Organization (NEDO) of Japan, and Okinawa prefecture.

\section{Author details}

${ }^{1}$ Departments of Pathology, Yamaguchi University Graduate School of Medicine, Ube-shi 755-8505, Japan. ${ }^{2}$ Departments of Surgery, Yamaguchi University Graduate School of Medicine, Ube-shi 755-8505, Japan. ${ }^{3}$ Division of Breast Disease Surgery, Shimonoseki Kohsei Hospital, Shimonoseki-shi 750-0061, Japan.

\section{Authors' contributions}

MY and KS designed and conducted the study. Kl, TF, SK and AO helped with the assays. SY and MO gathered tumor tissue specimens and clinicopathological data under a code of ethical conduct, MY drafted the manuscript. YN analyzed clinical data. KS conceived of this study, and he participated in its design and coordination. All the authors read and approved the final manuscript.

Received: 14 December 2011 Accepted: 25 July 2012

Published: 25 July 2012

\section{References}

1. Reis-Filho JS, Simpson PT, Gale T, Lakhani SR: Molecular evolution of breast cancer. J Pathol 2005, 205:248-254.

2. Oakman C, Bessi S, Zatarana E, Galardi F, Biganzoli L, Di Leo A: Recent adavances in systemic therapy new diagnostics and biological predictors of outcome in early breast cancer. Breast Cancer Res 2009, 11:205.

3. Rose ANN, Siegel PM: Emerging therapeutic targets in breast cancer bone metastasis. Future Oncol 2010, 6:55-74.

4. Raica M, Jung I, Cimpean AM, Suciu C, Muresan AM: From conventional pathologic diagnosis to the molecular classification of breast carcinoma: are we ready for the change? Rpm J Mrphol Embryol 2009, 50:5-13.

5. Goldhirsch A, Wood WC, Coates AS, Gelber RD, Thürlimann B, Senn HJ: Panel members. Strategies for subtypes--dealing with the diversity of breast cancer: highlights of the St. Gallen international expert consensus on the primary therapy of early breast cancer 2011. Ann Oncol 2011, 22:1736-1747.

6. Cheang MC, Voduc D, Bajdik C, Leung S, McKinney S, Chia SK, Perou CM, Nielsen TO: Basal-like breast cancer defined by five biomarkers has superior prgnostic value than triple-negative phenotype. Clin Cancer Res 2008, 14:1368-1376.

7. Tang P, Skinner KA, Hicks DG: Molecular classification of breast carcinomas by immunohistochemical analysis: are we ready? Diagn $\mathrm{Mol}$ Pathol 2009, 18:125-132.

8. Bayani J, Selvarajah S, Maire G, Vukovic B, Al-Romaih K, Zielenska M, Squire JA: Genomic mechanisms and measurement of structural and numerical instability in cancer cells. Semin Cancer Biol 2007, 17:5-18.

9. Haroske G, Baak JP, Danielsen H, Giroud F, Gschwendtner A, Oberholzer M, Reith A, Spieler P, Böcking A: Fourth updated ESACP consensus report on diagnostic DNA image cytometry. Anal Cell Pathol 2001, 23:89-95.

10. Millot C, Dufer J: Clinical applications of image cytometry to human tumour analysis. Histol Histopathol 2000, 15:1185-2000.

11. Suehiro Y, Okada T, Okada T, Anno K, Okayama N, Ueno K, Hiura M, Nakamura M, Kondo T, Oga A, Kawauchi S, Hirabayashi K, Numa F, Ito T, Saito T, Sasaki K, Hinoda Y: Aneuploidy predicts outcome in patients with endometrial carcinoma and is related to lack of $\mathrm{CDH} 13$ hypermethylation. Clin Cancer Res 2008, 14:3354-3361.

12. Abdalla FB, Boder JM, Buhmeida A, Elzaagheid A, Collan Y: Image DNA cytometry in FNABs of Libyan breast disease. Anticancer Res 2010, 30:175-181.

13. Yildirim Assaf S, Coumbos A, Hopfenmüller W, Foss H-D, Stein H, Kühn W: The prognostic significance of determining DNA content in breast cancer by DNA image cytometry: the role of high grade aneuploidy in node negative breast cancer. J Clin Pathol 2007, 60:649-655.

14. Ikemoto K, Furuya T, Oga A, Kawauchi S, Sasaki K: Relation of invasive ductal carcinoma by chromosome instability and breast cancer-related markers. J Jpn Soc Clin Cytol 2007, 46:332-337.

15. Umemura S, Kurosumi M, Moriya T, Oyama T, Arihiro K, Yamashita H, Umekita Y, Komoike Y, Shimizu C, Fukushima H, Kajiwara H, Akiyama F: Immunohistochemical evaluation for hormone receptors in breast cancer: a practically useful evaluation system and handling protocol. Breast Cancer 2006, 13:232-235.

16. Hammond ME, Hayes DF, Wolff AC, Mangu PB, Temin S: American society of clinical oncology/college of american pathologists guideline recommendations for immunohistochemical testing of estrogen and progesterone receptors in breast cancer. J Oncol Pract 2010, 6:195-197.

17. Cheang MC, Chia SK, Voduc D, Gao D, Leung S, Snider J, Watson M, Davies S, Bernard PS, Parker JS, Perou CM, Ellis MJ, Nielsen TO: Ki67 index, HER2 status, and prognosis of patients with luminal B breast cancer. J Natl Cancer Inst 2009, 101:736-750. 
18. Penault-Llorca F, André F, Sagan C, Lacroix-Triki M, Denoux $Y$, Verriele V, Jacquemier J, Baranzelli MC, Bibeau F, Antoine M, Lagarde N, Martin AL, Asselain B, Roché $\mathrm{H}$ : Ki67 expression and docetaxel efficacy in patients with estrogen receptor-positive breast cancer. J Clin Oncol 2009, 27:2809-2815

19. Colleoni M, Bagnardi V, Rotmensz N, Viale G, Mastropasqua M, Veronesi P, Cardillo A, Torrisi R, Luini A, Goldhirsch A: A nomogram based on the expression of $\mathrm{Ki}-67$, steroid hormone receptors status and number of chemotherapy courses to predict pathological complete remission after preoperative chemotherapy for breast cancer. Eur J Cancer 2010, 46:2216-2224

20. Miyazaki M, Furuya T, Shiraki A, et al: The Relationship of DNA ploidy to chromosomal Instability in primary human colorectal cancers. Cancer Res 1999, 59:5283-5285

21. Furuya T, Uchiyama T, Murakami T, Sato T, Oga A, Sasaki K: Relationship between chromosomal instability and intratumoral regional DNA ploidy heterogeneity in primary gastric cancers. Clin Cancer Res 2000 6:2815-2820.

22. Yamamoto $Y$, Matsuyama $H$, Kawauchi $\mathrm{S}$, et al: Biological characteristics in bladder cancer depend on the type of genetic instability. Clin Cancer Res 2006, 12:2752-2758.

23. Sasaki K, Matsumura K, Murakami T, Tsuji T: Measurement of bromodeoxyuridine labeling index, Ki-67 score and Ag-NOR count in breast carcinomas. Comparison with DNA ploidy. Oncology 1992, 49:147-153.

24. Sasaki K, Matsumura K, Tsuji T, Shinozaki F, Takahashi M: Relationship between labeling indices of Ki-67 and BrdUrd in human malignant tumors. Cancer 1988, 62:989-993.

25. Voduc KD, Cheang MC, Tydesley S, Geimon K, Nielsen TO, Kennecke H: Breast cancer subtypes and the risk of local and regional relapse. J Clin Oncol 2010, 28:1684-1691.

26. Lengauer C, Kinzler KW, Vogelstein B: Genetic instability in colorectal cancers. Nature 1997, 386:623-627.

27. Lengauer C, Kinzler KW, Vogelstein B: Genetic instabilities in huma cancers. Nature 1998, 396:643-649.

28. Kawauchi S, Furuya T, Ikemoto K, Nakao M, Yamamoto S, Oka M, Sasaki K: DNA copy number aberrations associated with aneuploidy and chromosomal instability in breast cancers. Oncol Rep 2010, 24:875-883.

29. Smid M, Hoes M, Sieuwerts AM, Sleifer S, Zhang Y, Wang Y, Foekens JA, Martens JW: Patterns and incidence of chromosomal instability and their prognostic relevance in breast cancer subtypes. Breast Cancer Res Treat 2011, 128:23-30.

30. Li L, Mu K, Zhou G, Lan L, Auer G, Zetterberg A: Genomic instability and proliferative activity as risk factors for distant metastases in breast cancer. Br J Cancer 2008, 99:513-519.

31. Williams BR, Amon A: Aneuploidy-cancer's fatall flaw? Cancer Res 2009, 69:5289-5291.

32. Toft DJ, Cryns VL: Minireview: basal-like breast cancer: from molecular profiles to targeted therapies. Mol Endocrinol 2011, 25:199-211.

33. Carter SL, Eklund AC, Kohane IS, Harris LN, Szallasi Z: A signature of chromosomal instability inferred from gene expression profiles predicts clinical outcome in multiple human cancers. Nat Genet 2006, 38:1043-1048.

34. Habermann JK, Doering J, Hautaniemi S, Roblick UJ, Bündgen NK, Nicorici D, Kronenwett U, Rathnagiriswaran S, Mettu RK, Ma Y, Krüger S, Bruch HP, Auer G, Guo NL, Ried T: The gene expression signature of genomic instability in breast cancer is an independent predictor of clinical outcome. Int $J$ Cancer 2009, 124:1552-1564.

35. Birkbak NJ, Eklund AC, Li Q, McClelland SE, Endesfelder D, Tan P, Tan IB, Richardson AL, Szallasi Z, Swanton C: Paradoxical relationship between chromosomal instability and survival outcome in cancer. Cancer Res 2011, 71:3447-3452.

36. Roylance R, Endesfelder D, Gorman P, Burrell RA, Sander J, Tomlinson I, Hanby AM, Speirs V, Richardson AL, Birkbak NJ, Eklund AC, Downward J, Kschischo M, Szallasi Z, Swanton C: Relationship of extreme chromosomal instability with long-term survival in a retrospective analysis of primary breast cancer. Cancer Epidemiol Biomarkers Prev 2011, 20:2183-2194.

37. Fitzgibbons PL, Page DL, Weaver D, Thor AD, Allred DC, Clark GM, Ruby SG, O'Malley F, Simpson JF, Connolly JL, Hayes DF, Edge SB, Lichter A, Schnitt

$\mathrm{SJ}$ : Prognostic factors in breast cancer. College of American Pathologists Consensus Statement 1999. Arch Pathol Lab Med 2000, 124:966-978.

doi:10.1186/1756-0500-5-376

Cite this article as: Yanagawa et al:: Luminal A and luminal B (HER2 negative) subtypes of breast cancer consist of a mixture of tumors with different genotype. BMC Research Notes 2012 5:376.

\section{Submit your next manuscript to BioMed Central and take full advantage of:}

- Convenient online submission

- Thorough peer review

- No space constraints or color figure charges

- Immediate publication on acceptance

- Inclusion in PubMed, CAS, Scopus and Google Scholar

- Research which is freely available for redistribution 